\title{
Retraction
}

\section{Retracted: Increasing the Stimulation Dose of rFSH in Unexpected Poor Responders Is Not Associated with Better IVF Outcome}

\author{
The Scientific World Journal \\ Received 11 October 2012; Accepted 11 October 2012 \\ Copyright (C) 2012 The Scientific World Journal. This is an open access article distributed under the Creative Commons Attribution \\ License, which permits unrestricted use, distribution, and reproduction in any medium, provided the original work is properly \\ cited.
}

This article has been retracted as it was found to contain falsified data. Additionally, it was found that the same article was published in Ginekologia Polska (2012, 83, 2, 111-115) [1].

\section{References}

[1] L. Tutuncu and O. Dundar, "Increasing the stimulation dose of $\mathrm{rFSH}$ in unexpected poor responders is not associated with better IVF outcome," The Scientific World Journal, vol. 2012, Article ID 202804, 5 pages, 2012. 


\section{Clinical Study}

\section{Increasing the Stimulation Dose of rFSH in Unexpected Poor Responders Is Not Associated with Better IVF Outcome}

\section{Levent Tutuncu and Ozgur Dundar}

IVF Unit, Department of Obstetrics and Gynecology, Haydarpasa Training Hospital, Gulhane Military Medical Academy, Haydarpasa, 34668 Istanbul, Turkey

Correspondence should be addressed to Levent Tutuncu, ltutuncu@yahoo.com

Received 11 October 2011; Accepted 14 December 2011

Academic Editor: Jose G. Cecatti

Copyright $\odot 2012$ L. Tutuncu and O. Dundar. This is an open access article distributed under the Creative Commons Attribution License, which permits unrestricted use, distribution, and reproduction in any medium, provided the original work is properly cited.

The aim of this retrospective study is to determine whether increasing the stimulation dose of rFSH in unexpected poor responders is associated with better in vitro fertilization (IVF) outcome or not. A total of forty eligible women who fulfilled our definition of poor responders and who did not achieve an ongoing pregnancy in the first cycle and returned for a second higher $\mathrm{rFSH}$ dose IVF cycle with a long-agonist protocol were included to the study. The first low-dose cycles and the second high-dose cycles were compared to each other. Main outcome measures of the study were duration of stimulation, number of follicles, number of oocytes retrieved, number of embryos, and E2 level on day of hCG injection. There were no significant differences in duration of stimulation, number of follicles, number of oocytes retrieved, number of embryos, and E2 level on day of hCG injection between the first low- and second high-dose cycles. Daily dose and total dose of rFSH were significantly higher in the second high-dose cycles. Increasing the dose of rFSH in a second stimulation cycle after first unsuccessful treatment cycle will add only to the cost and discomfort of the treatment and might adversely affect pregnancy rates.

\section{Introduction}

The primary aim of in vitro fertilization (IVF) treatment is the birth of a healthy baby. For the purpose of IVF, controlled ovarian hyperstimulation $(\mathrm{COH})$ is performed by using exogenous follicle stimulating hormone (FSH) to generate as many follicles as possible and to maximize the chances of producing good quality embryos available for transfer. But some patients respond poorly during $\mathrm{COH}$ and poor response to $\mathrm{COH}$ leads to a low number of oocytes and fewer embryos and as a result poor response associates with high cancellation rates and low pregnancy rates. The incidence of poor response to $\mathrm{COH}$ has been reported between $9 \%$ and $24 \%$ [1].

The starting daily dose of gonadotrophin is generally based on the patient's age, basal FSH level, and eventually response to prior stimulation protocols. At present the ideal stimulation regimen for poor responders is not known and age may not be an important factor [2]. It is generally believed that older and poor responder patients require higher doses to overcome the decline in ovarian response. But this practice is based on empirical evidence rather than on sound evidence [3]. Although increasing doses of gonadotrophins have been shown to increase the number of collected oocytes [4], later this improvement was explained by a phenomenon called "regression to the mean" [5] and increasing the dose of FSH above a threshold dose had not been found valuable [6], even if it may have adverse implications on pregnancy rates [7]. Moreover, it has been shown that an unexpected poor response in a young patient in the first IVF cycle was not necessarily related to a poor prognosis in subsequent cycles [8]. Ovarian sensitivity for gonadotrophins varies among women, and an individualized approach is most likely to result in a sufficient number of oocytes with minimal side effects [9].

The objective of this study is to observe the second higher gonadotrophin dose IVF outcome in unexpected poor responder patients. In particular we investigated if a high daily dose of $\mathrm{rFSH}$ in poor responders is associated with higher number of oocytes at the time of retrieval and improved 
TABLE 1: Baseline information on the characteristics of women and regimens.

\begin{tabular}{|c|c|}
\hline Parameter & First cycle (low dose) \\
\hline Age $(\text { years) })^{\mathrm{a}}$ & $34.35 \pm 3.88$ \\
\hline Basal FSH $(\mathrm{mIU} / \mathrm{mL})$ on day $3^{\mathrm{a}}$ & $7.3 \pm 1.91$ \\
\hline BMI $\left(\mathrm{kg} / \mathrm{m}^{2}\right)^{\mathrm{a}}$ & $22.3 \pm 3.1$ \\
\hline Treatment duration (days) ${ }^{\mathrm{a}}$ & $11.45 \pm 1.67$ \\
\hline E2 level on day of HCG $(\mathrm{pg} / \mathrm{mL})^{\mathrm{a}}$ & $711.8 \pm 278.4$ \\
\hline Total rFSH dose (IU) $)^{\mathrm{a}}$ & $2018.06 \pm 557.6$ \\
\hline rFSH dose (IU) per stimulation day ${ }^{\mathrm{a}}$ & $176.25 \pm 36.22$ \\
\hline
\end{tabular}

Values are mean $\pm \mathrm{SD}$

NS = Not significant.

pregnancy rate. We analyzed retrospectively IVF data from May 2007 to May 2011 for patients who had at least two cycles of stimulation with rFSH and a higher dose in second cycle because of an unexpected poor response in the first cycle.

\section{Methods}

We reviewed IVF cycles performed at the Gulhane Military Medical Academy, Haydarpasa Training Hospital IVF Unit, from May 2007 to May 2011 retrospectively. Patient charts were reviewed and the following data recorded: age, body mass index (BMI), cause of infertility, basal day 3 serum FSH value, total amount of rFSH dose in IU used during the stimulation, cycle length, E2 serum value on day of hCG, number of follicles $\geq 17 \mathrm{~mm}$ on day of hCG, number of oocytes retrieved, and number of transferable embryos. General medical and gynecological histories were recorded together with the result of physical examination and laboratory testing.

Patients were included in the study if they had completed a stimulation protocol with rFSH (Gonal-F, Merck Serono, Turkey, or Puregon, Schering-Plough, Turkey) and fulfilled our standard definition of poor responders and who did not achieve an ongoing pregnancy in the first cycle and who returned for a second higher-dose IVF cycle. Although different criteria were used in various published studies and lack a uniform definition of poor response, we defined poor responders as patients with less than 4 mature oocytes retrieved and less than or equal to 3 follicles and/or Estradiol (E2) levels of less than $500 \mathrm{pg} / \mathrm{mL}$ on the day of hCG administration. ESHRE recently presented "a consensus paper" to standardize the definition of poor response and they proposed similar criteria [10]. All patients who were eligible for the study agreed to participate and each patient provided their written informed consent.

All patients started with 150-225 IU per day with $\mathrm{rFSH}$ s.c. injection on the first cycle (first low-dose cycle) and 225375 IU per day in a consecutive next cycle (second high-dose cycle). The second high-dose stimulation cycle was within 1 year after first low-dose stimulation cycle. Other inclusion criteria include the following: age $<40$ years and BMI $\leq$ $29 \mathrm{~kg} / \mathrm{m}^{2}$ or $>18 \mathrm{~kg} / \mathrm{m}^{2}, \mathrm{FSH} \leq 12 \mathrm{IU} / \mathrm{L}$, regular spontaneous menstrual cycle (24-35 days) and presence of two ovaries.
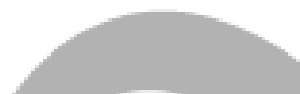
TABLE 2: Comparison of two consecutive cycles which dose of rFSH has been increased.

\begin{tabular}{lccc}
\hline Parameter & $\begin{array}{c}\text { First cycle } \\
\text { (low dose) }\end{array}$ & $\begin{array}{c}\text { Second cycle } \\
\text { (high dose) }\end{array}$ & $P$ value \\
\hline $\begin{array}{l}\text { Number of aspirated } \\
\text { follicles }^{\mathrm{a}}\end{array}$ & $2.30 \pm 0.64$ & $2.375 \pm 0.66$ & $\mathrm{NS}$ \\
$\begin{array}{l}\text { Number of oocytes } \\
\text { retrieved }\end{array}$ & $1.40 \pm 1.15$ & $1.50 \pm 0.877$ & $\mathrm{NS}$ \\
$\begin{array}{l}\text { Fertilization rate }(\%) \\
\begin{array}{l}\text { Number of transferable } \\
\text { embryos }\end{array}\end{array}$ & 55.3 & 56.6 & $\mathrm{NS}$ \\
Cancelled cycles $^{\mathrm{a}}$ & $12 / 40=30 \%$ & $5 / 40=12.5 \%$ & $P<0.05$ \\
\hline
\end{tabular}

${ }^{\mathrm{a}}$ Values are mean $\pm \mathrm{SD}$

NS $=$ Not significant.

$711.8 \pm 278.4 \mathrm{pg} / \mathrm{mL}$ and $822.42 \pm 311.2 \mathrm{pg} / \mathrm{mL}$ in first lowdose cycle and second high-dose cycle, respectively, and there was no significant difference $(P \geq 0.05)$.

At first low-dose cycle $70 \%$ of the patients had oocyte retrieval and $55 \%$ had embryo transfer. On the other hand at second high-dose cycle oocyte retrieval and embryo transfer rates were significantly higher than those of the first low-dose cycle $(87.5 \%$ and $62.5 \%$, resp. $)(P<0.05)$. But the outcome measures were not different between the first low-dose cycle and second high-dose cycle (Table 2). Patients showed no significant difference in mean number of follicles $(2.30 \pm 0.64$ versus $2.375 \pm 0.66)$ and mean number of oocytes retrieved $(1.40 \pm 1.15$ versus $1.50 \pm 0.877)(P \geq 0.05)$. Upward FSH dose adjustment did not produce any significant increase in the mean number of transferable embryos per cycle $(0.775 \pm$ 0.8 versus $0.85 \pm 0.8, P \geq 0.05)$. Fertilization rates were similar in the first low-dose cycle and second high-dose cycle (55.3\% and 56.6\%, resp.) $(P \geq 0.05)$.

Cycle lengths were similar in the 2 groups $(11.45 \pm 1.67$ days versus $11.7 \pm 1.11$ days). But $\mathrm{rFSH}$ dose (IU) per stimulation day $(176.25 \pm 36.22 \mathrm{IU}$ versus $285.00 \pm 59.32 \mathrm{IU})$ and the total dose of gonadotrophins used (2018.06 \pm $557.6 \mathrm{IU}$ versus $3334.5 \pm 643.2 \mathrm{IU}$ ) were significantly higher in the second high-dose cycle group compared with the first low-dose group $(P<0.05)$, with higher cost in the second high-dose group.

More cycles were cancelled during the low-dose stimulation protocol $(12 / 40=30 \%$ versus $5 / 40=12.5 \%, P<0.05)$. We did not analyze the pregnancy rates on the first low-dose cycle because the patients who become pregnant after first cycle were not included in the study. However there were only 2 pregnancies after second high dose cycle. Ongoing pregnancy rate in the second high-dose cycle was only $5 \%$. Compared to a historic normal responder control group this represents a very low percentage (data not shown).

There was no ovarian hyperstimulation or hospitalization for any other reason during the treatment period.

\section{Discussion}

In this retrospective study we demonstrated that increasing the starting dose of gonadotrophins after an unexpected poor response in the first IVF cycle reduces the cancellation rate. Besides it may increase the oocyte retrieval rates and embryo transfer rates. But increasing the dose of gonadotrophin in a second cycle after the first unexpected poor response did not increase the number of oocytes retrieved and the number of transferable embryos.

A generally accepted practice in IVF is to increase the daily dose of gonadotrophins if the first treatment cycle is unsuccessful. This is done with the belief that a higher gonadotrophin dose will stimulate a somehow compromised ovary to a better response, assuming a dose response relationship between gonadotrophins administered and E2 levels and number of follicles. But if this relationship is true for good responders, the situation is physiologically different in poor responders. This study gives empirical evidence to this statement. The ovary of poor responders (although with a normal FSH levels) is less responsive to exogenous gonadotrophins. This underlines the poor predictive value of day $3 \mathrm{FSH}$ in this subgroup of patients and it suggests that there may be other reasons than reduced ovarian reserve for a poor response.

During ovulation induction for assisted reproductive technologies, increasing the number of oocytes collected can allow better embryo selection and maybe better pregnancy rates [11]. Several authors analyzed the effect of increasing the gonadotrophins stimulation dose to poor responders. Initial studies were performed with purified urinary FSH. In 1989, Hofmann et al. showed that ovarian hyperstimulation with high doses of FSH may result improved E2 response and fewer cancellations in poor responder patients, and they suggested that high-dose FSH may offer a good alternative for these patients [12]. But only one year later in another study from the same center, Karande et al. concluded that high-dose FSH stimulation at the onset of the menstrual cycle does not improve the IVF outcome in low-responder patients [13]. Land et al. doubled the FSH dose in the second cycle to improve the outcome in poor responders [14]. They reported a significant increase in the number of oocytes but not in the number of embryos. Lashen et al. [4] conducted a retrospective study and reported that low- and average-responder patients but not high-responder patients had significantly higher numbers of eggs, embryos, and higher E2 levels in their second cycles in which the dose of gonadotrophin was increased. But they found that there was no difference for the pregnancy rate between the groups although there were differences for other parameters. They suggested the existence of a maximum effective dose for this preparation. They found that increasing the FSH dose above $300 \mathrm{IU} /$ day after a poor response in the first cycle is pointless. However, Pantos et al. showed that increasing the gonadotrophin dose above $150 \mathrm{IU} /$ day did not increase the number of oocytes in poor responders [15]. In a prospective randomized trial of Garcia-Velasco et al., early cessation of GnRHa combined with high doses of gonadotrophins had recruited a significantly higher number of mature oocytes but resulted in similar IVF outcome and pregnancy rates than a conventional nonstop, long downregulation protocol [16].

More recent studies analyzed the same questions using rFSH. Studies comparing different doses of rFSH suggest 
that pregnancy rates do not increase with increasing doses of rFSH. Two different randomized double-blind clinical trials are analyzed if a higher-dose protocol is associated with better outcome. Fixed daily doses of recombinant FSH (rFSH) (100 versus $200 \mathrm{IU})$ resulted in higher number of oocytes at the time of the retrieval in the high-dose group, but the clinical pregnancy rates were the same and more sideeffects were noted with the high-dose regimen $[17,18]$. Another study showed that an increase in $\mathrm{rFSH}$ daily dose from 150 IU to 250 IU did not result in a higher number of retrievable oocytes in older women [19]. In a randomized, double-blind multicenter clinical trial, the Latin American Puregon study concluded that an increase in the daily recombinant FSH dose from 150 to $250 \mathrm{IU}$ in women between 30 and 39 years of age has only limited benefit, and the clinical use of these higher doses can be questioned [20]. The main difference between these trials [17-20] and our study is that we followed the same group of patients in a second high-dose cycle; therefore each patient functioned as her own control.

In a systematic review, Tarlatzis et al. [21] showed that the results of the trials were controversial, and there is no evidence supporting the use of high-doses of gonadotrophins in poor responders. In a prospective randomized controlled study, expected poor responders on the basis of an antral follicle count did not benefit from a higher starting dose of gonadotrophins in IVF treatment with a daily dosage of $300 \mathrm{IU}$ of recombinant FSH when compared with $150 \mathrm{IU}$ [22]. Lekamge et al. [6] reported the same findings in a retrospective study. Our results are very similar with these studies. Siristatidis and Hamilton [23] had evaluated the clinical trials about the effectiveness of the high dose FSH regimes and tried to find out the safest and most effective upper limit of FSH that might be used for $\mathrm{COH}$ in poor responders. They concluded that most of the retrospective studies and reviews had showed that to increase the dose was useless. But their comments about some prospective randomized trials were quite interesting, because they had given particulars a satisfactory ovarian response and pregnancy rate would achieved when the FSH dose exceed the $300 \mathrm{IU}$ dosage, being combined with various types of downregulation or adjuvant strategies. A recent metaanalysis suggested that the optimal daily rFSH stimulation dose is $150 \mathrm{IU} /$ day in presumed normal responders younger than 39 years undergoing IVF [24]. In this study the average number of oocytes retrieved per pick-up was increased when higher $\mathrm{rFSH}$ doses over $100 \mathrm{IU} /$ day were given, whereas pregnancy rates did not differ across the dosage range tested and cumulative pregnancy rates including additional cryo embryo transfer cycles would not become superior with dosages exceeding $150 \mathrm{IU} /$ day. The conclusion of this metaanalysis was when using $150 \mathrm{IU} /$ day the probability of poor response was not different from higher dosages, indicating that $150 \mathrm{IU} /$ day is likely to represent the optimal dosage in general. Our results confirm these findings. Despite receiving higher daily, and total rFSH doses in the second treatment cycles, the numbers of follicles, the number of oocytes retrieved and total number of transferable embryos were all similar in both the low-dose and high-dose treatment cycles.
Nowadays, mild ovarian stimulation protocols for IVF are being developed in order to reduce the cost and to minimize the complications of $\mathrm{COH}$. Other benefits of mild stimulation might be the probability of generating better embryos and more receptive endometrium because of the potentially negative effects of supraphysiological steroid levels on oocyte quality and endometrial receptivity [25]. Although the usual mild stimulation protocol is held with $\mathrm{GnRH}$ antagonists, mild IVF using GnRHa long protocol is possible too [26]. But, mild ovarian stimulation has not yet been tested in the context of ovarian aging [27]. After mild ovarian stimulation, a modest number of oocytes are associated with optimal implantation rates and do not reflect a poor ovarian response [28]. When we evaluate and compare our results with these data about mild ovarian stimulation, increasing the dose of rFSH in a second stimulation cycle after first unsuccessful treatment cycle will add only to the cost and discomfort of the treatment and may adversely effect pregnancy rates.

\section{Conclusion}

The results of our study indicate that increasing the starting dose of gonadotrophin after an unexpected poor response in the first IVF cycle is not an effective approach. It may increase the oocyte retrieval rates and embryo transfer rates but will not add any significant improvement in the number of oocytes retrieved and the number of transferable embryos. The only important benefit of increasing the dose was the low cancellation rate. There is no linear relationship between the dose of recombinant FSH and the ovarian response in unexpected poor responders.

\section{Conflict of Interests}

The authors declare that they have no conflict of interests.

\section{References}

[1] D. Kyrou, E. M. Kolibianakis, C. A. Venetis, E. G. Papanikolaou, J. Bontis, and B. C. Tarlatzis, "How to improve the probability of pregnancy in poor responders undergoing in vitro fertilization: a systematic review and meta-analysis," Fertility and Sterility, vol. 91, no. 3, pp. 749-766, 2009.

[2] B. Vollenhoven, T. Osianlis, and J. Catt, "Is there an ideal stimulation regimen for IVF for poor responders and does it change with age?" Journal of Assisted Reproduction and Genetics, vol. 25, no. 11-12, pp. 523-529, 2008.

[3] M. H. A. Van Hooff, "The human menopausal gonadotropin (hMG) dose in in vitro fertilization (IVF): what is the optimal dose?" Journal of Assisted Reproduction and Genetics, vol. 12, no. 4, pp. 233-235, 1995.

[4] H. Lashen, W. Ledger, Bernal A. López, B. Evans, and D. Barlow, "Superovulation with a high gonadotrophin dose for in vitro fertilization: is it effective?" Journal of Assisted Reproduction and Genetics, vol. 15, no. 7, pp. 438-443, 1998.

[5] L. Rombauts, "Is there a recommended maximum starting dose of FSH in IVF?" Journal of Assisted Reproduction and Genetics, vol. 24, no. 8, pp. 343-349, 2007. 
[6] D. N. Lekamge, M. Lane, R. B. Gilchrist, and K. P. Tremellen, "Increased gonadotrophin stimulation does not improve IVF outcomes in patients with predicted poor ovarian reserve," Journal of Assisted Reproduction and Genetics, vol. 25, no. 1112, pp. 515-521, 2008.

[7] L. Pal, S. Jindal, B. R. Witt, and N. Santoro, "Less is more: increased gonadotropin use for ovarian stimulation adversely influences clinical pregnancy and live birth after in vitro fertilization," Fertility and Sterility, vol. 89, no. 6, pp. 1694-1701, 2008.

[8] E. R. Klinkert, F. J. M. Broekmans, C. W. N. Looman, and E. R. Te Velde, "A poor response in the first in vitro fertilization cycle is not necessarily related to a poor prognosis in subsequent cycles," Fertility and Sterility, vol. 81, no. 5, pp. 1247-1253, 2004.

[9] B. Popovic-Todorovic, A. Loft, S. Ziebe, and A. Nyboe Andersen, "Impact of recombinant FSH dose adjustments on ovarian response in the second treatment cycle with IVF or ICSI in "standard" patients treated with 150 IU/day during the first cycle," Acta Obstetricia et Gynecologica Scandinavica, vol. 83, no. 9, pp. 842-849, 2004.

[10] A. P. Ferraretti, A. La Marca, B. C. J. M. Fauser, B. Tarlatzis, G. Nargund, and L. Gianaroli, "ESHRE consensus on the definition of "poor response" to ovarian stimulation for in vitro fertilization: the Bologna criteria," Human Reproduction, vol. 26, no. 7, pp. 1616-1624, 2011.

[11] N. S. Macklon, R. L. Stouffer, L. C. Giudice, and B. C. J. M. Fauser, "The science behind 25 years of ovarian stimulation for in vitro fertilization," Endocrine Reviews, vol. 27, no. 2, pp. 170-207, 2006.

[12] G. E. Hofmann, J. P. Toner, S. J. Muasher, and G. S. Jones, "High-dose follicle-stimulating hormone (FSH) ovarian stimulation in low-responder patients for in vitro fertilization," Journal of In Vitro Fertilization and Embryo Transfer, vol. 6, no. 5, pp. 285-289, 1989.

[13] V. C. Karande, G. S. Jones, L. L. Veeck, and S. J. Muasher, "High-dose follicle-stimulating hormone stimulation at the onset of the menstrual cycle does not improve the in vitro fertilization outcome in low-responder patients," Fertility and Sterility, vol. 53, no. 3, pp. 486-489, 1990.

[14] J. A. Land, M. I. Yarmolinskaya, J. C. M. Dumoulin, and J. L. H. Evers, "High-dose human menopausal gonadotropin stimulation in poor responders does not improve in vitro fertilization outcome," Fertility and Sterility, vol. 65, no. 5, pp. 961965, 1996.

[15] C. Pantos, S. J. Thornton, A. L. Speirs, and I. Johnston, "Increasing the human menopausal gonadotropin dose - does the response really improve?" Fertility and Sterility, vol. 53, no. 3, pp. 436-439, 1990.

[16] J. A. Garcia-Velasco, V. Isaza, A. Requena et al., "High doses of gonadotrophins combined with stop versus non-stop protocol of GnRH analogue administration in low responder IVF patients: a prospective, randomized, controlled trial," Human Reproduction, vol. 15, no. 11, pp. 2292-2296, 2000.

[17] H. J. Out, S. Lindenberg, A. L. Mikkelsen et al., "A prospective, randomized, double-blind clinical trial to study the efficacy and efficiency of a fixed dose of recombinant follicle stimulating hormone (Puregon(TM)) in women undergoing ovarian stimulation," Human Reproduction, vol. 14, no. 3, pp. 622-627, 1999.

[18] H. J. Out, I. David, R. Ron-El et al., "A randomized, doubleblind clinical trial using fixed daily doses of 100 or 200 IU of recombinant FSH in ICSI cycles," Human Reproduction, vol. 16, no. 6, pp. 1104-1109, 2001.
[19] H. J. Out, D. D. M. Braat, B. M. E. Lintsen, T. Gurkan, O. Bukulmez, O. Gokmen et al., "Increasing the daily dose of recombinant follicle stimulating hormone (puregon) does not compensate for age-related decline in retrievable oocytes after controlled ovarian hyperstimulation," Human Reproduction, vol. 15, pp. 29-35, 2000.

[20] H. J. Out, "A double-blind clinical trial comparing a fixed daily dose of 150 and 250 IU of recombinant follicle-stimulating hormone in women undergoing in vitro fertilization," Fertility and Sterility, vol. 76, no. 5, pp. 950-956, 2001.

[21] B. C. Tarlatzis, L. Zepiridis, G. Grimbizis, and J. Bontis, "Clinical management of low ovarian response to stimulation for IVF: a systematic review," Human Reproduction Update, vol. 9, no. 1, pp. 61-76, 2003.

[22] E. R. Klinkert, F. J. M. Broekmans, C. W. N. Looman, J. D. F. Habbema, and E. R. te Velde, "Expected poor responders on the basis of an antral follicle count do not benefit from a higher starting dose of gonadotrophins in IVF treatment: a randomized controlled trial," Human Reproduction, vol. 20, no. 3, pp. 611-615, 2005.

[23] C. S. Siristatidis and M. P. Hamilton, "What should be the maximum FSH dose in IVF/ICSI in poor responders?" Journal of Obstetrics and Gynaecology, vol. 27, no. 4, pp. 401-405, 2007.

[24] M. D. Sterrenburg, S. M. Veltman-Verhulst, M. J.C. Eijkemans et al., "Clinical outcomes in relation to the daily dose of recombinant follicle-stimulating hormone for ovarian stimulation in in vitro fertilization in presumed normal responders younger than 39 years: a meta-analysis," Human Reproduction Update, vol. 17, no. 2, pp. 184-196, 2011.

[25] M. F. G. Verberg, N. S. Macklon, G. Nargund et al., "Mild ovarian stimulation for IVF," Human Reproduction Update, vol. 15, no. 1, pp. 13-29, 2009.

[26] S. Fernández-Shaw, N. Pérez Esturo, R. Cercas Duque, and I. Pons Mallol, "Mild IVF using GnRH agonist long protocol is possible: comparing stimulations with $100 \mathrm{IU}$ vs. $150 \mathrm{IU}$ recombinant FSH as starting dose," Journal of Assisted Reproduction and Genetics, vol. 26, no. 2-3, pp. 75-82, 2009.

[27] B. C. J. M. Fauser, G. Nargund, A. N. Andersen et al., "Mild ovarian stimulation for IVF: 10 years later," Human Reproduction, vol. 25, no. 11, pp. 2678-2684, 2010.

[28] M. F. G. Verberg, M. J. C. Eijkemans, N. S. Macklon et al., "The clinical significance of the retrieval of a low number of oocytes following mild ovarian stimulation for IVF: a meta-analysis," Human Reproduction Update, vol. 15, no. 1, pp. 5-12, 2009. 